\title{
The Radiative Forcing of Aerosols in a West Africa Sahelian Urban City: Case Study of Ouagadougou
}

\author{
Bruno Korgo ${ }^{1 *}$, Pétronille Kafando ${ }^{2}$, Bernard Zouma ${ }^{1}$, Nebon Badoํㅜ, Issa Zerbo ${ }^{1}$, \\ Jean-Claude Roger ${ }^{3}$, Joseph D. Bathiebo ${ }^{1}$
}

${ }^{1}$ Laboratory of Thermal and Renewable Energy, Department of Physics, University Joseph KI-ZERBO, Ouagadougou, Burkina Faso

${ }^{2}$ Laboratory of Environmental Physics and Chemistry, Department of Physics, University Joseph KI-ZERBO, Ouagadougou, Burkina Faso

${ }^{3}$ Department of Geographical Sciences, University of Maryland, College Park, MD, USA

Email: ^brunokor@yahoo.fr

How to cite this paper: Korgo, B., Kafando, P., Zouma, B., Bado, N., Zerbo, I., Roger, J.-C. and Bathiebo, J.D. (2021) The Radiative Forcing of Aerosols in a West Africa Sahelian Urban City: Case Study of Ouagadougou. Atmospheric and Climate Sciences, 11, 73-85.

https://doi.org/10.4236/acs.2021.111005

Received: October 25, 2020

Accepted: January 1, 2021

Published: January 4, 2021

Copyright $\odot 2021$ by author(s) and Scientific Research Publishing Inc. This work is licensed under the Creative Commons Attribution International License (CC BY 4.0).

http://creativecommons.org/licenses/by/4.0/

\begin{abstract}
This paper is an assessment of radiative forcing caused by atmospheric aerosols in an urban city in West Africa. It is carried out in Ouagadougou in Burkina Faso and is an illustration of the radiative impact in most of the large Sahelian urban cities which are under the same climatic influences and whose populations present similarities in their socio-economic aspects. Using the GAME code, the radiative forcing was calculated at the top of the atmosphere, in the atmospheric layer and at the earth's surface. The results showed overall a cooling effect at the top of the atmosphere due to the backscattering in space of the incident radiation, a heating in the atmospheric layer due to the absorption effect and a surface cooling justified by the attenuation of radiation crossing the atmosphere. Using monthly average values of optical properties, vertical temperature and humidity profiles, daily temperatures and surface albedo, the simulation yielded forcing values ranging from -6.77 $\mathrm{W} / \mathrm{m}^{2}$ to $-2.56 \mathrm{~W} / \mathrm{m}^{2}$ at the top of the atmosphere, from $15.8 \mathrm{~W} / \mathrm{m}^{2}$ to 34.7 $\mathrm{W} / \mathrm{m}^{2}$ in the atmospheric layer and from $-41.00 \mathrm{~W} / \mathrm{m}^{2}$ to $-21.68 \mathrm{~W} / \mathrm{m}^{2}$ at the earth's surface. In addition, the warming was simulated in the first atmospheric layer (in contact with the surface), and the results show values ranging from $0.8^{\circ} \mathrm{C}$ to $1.8^{\circ} \mathrm{C}$. The study of the annual variability of the results showed a strong correlation between the radiative forcing and the seasonal succession characteristic of the climate in West Africa with the extreme values in the month of March (characteristic of the dry and hot season) and in the month of August (characteristic of the rainy season).
\end{abstract}




\section{Keywords}

Aerosols, Radiative Forcing, Scattering, Absorption, GAME Code, AERONET

\section{Introduction}

Atmospheric aerosols are known to have significant impacts on health, the environment and the climate. We attribute to aerosols, an important part of climate change whose harmful effects sow the misfortune of many populations in various parts of the world. At the climatic level, the effects of aerosols are categorized into three: direct impacts, semi-direct impacts and indirect impacts. The direct impact of aerosols results in the direct attenuation or intensification of solar or terrestrial radiation resulting from scattering [1] [2] or absorption [3]. The semi-direct impacts are due to certain aerosols which cause the warming of the troposphere with the consequence of modifying the temperature and humidity profile thus impacting the vertical balance of the atmosphere. This warming effect can inhibit the process of cloud formation or promote their evaporation [4]. The indirect effect is manifested in the presence of aerosols in the clouds, and they modify the optical properties, size and number of water droplets they contain. They thus lead to a modification of the entire hydrological cycle [5]. These effects are fully described by the "Twomey effect" [6] [7] [8] and the "AIbrecht effect" [9] [10].

It clearly appears that the radiative forcing caused by aerosols plays an important role in the climatic effects described above. This work aims to evaluate the radiative forcing caused by the aerosol population of the city of Ouagadougou using a version of the GAME code [11] modified by [12]. This city is the capital of Burkina Faso and located at the gateway to the Sahel in West Africa. Due to its geographical position, it is strongly impacted by the combined effect of Saharan desert dust and urban pollution linked to human activity [13]. We assess this radiative impact at three levels of the atmospheric column, namely the top of the atmosphere, the atmospheric layer and the earth's surface.

\section{The Radiative Transfer Equation}

Consider a radiation brush which crosses a surface element $\mathrm{d} \sigma$ at an angle of incidence $\vartheta$ and confined in an elementary solid angle $\mathrm{d} \omega$ (Figure 1).

The amount of energy $\mathrm{d} E_{v}$ transported through this surface during the time $\mathrm{d} t$, in a frequency interval between $v$ and $v+\mathrm{d} v$ is given by the following equation:

$$
\mathrm{d} E_{v}=I_{\nu} \cos \vartheta \mathrm{d} v \mathrm{~d} \omega \mathrm{d} t
$$

where $I_{v}$ denotes in this expression, the intensity of the radiation in the specified frequency band 


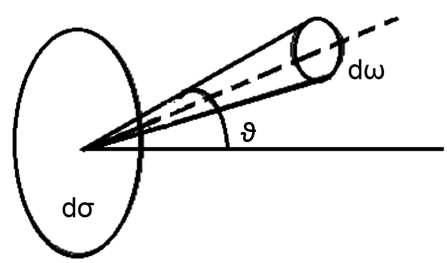

Figure 1. Illustration of incident radiation on a surface element.

The radiative transfer equation relates the intensity of a source radiation that encounters a particle or passes through a medium with the absorption, diffusion and possibly the emission that characterizes it.

\section{- The absorption component.}

The attenuation undergone by the radiation brush when crossing a medium is expressed by:

$$
\mathrm{d} I_{v}=-K_{v} \rho I_{v} \mathrm{~d} s
$$

where $K_{v}$ defines the mass absorption coefficient for a radiation of frequency $v, \rho$ the density of the medium. The corresponding energy loss is written:

$$
K_{v} \rho \mathrm{d} s \cdot I_{v} \cos \vartheta \mathrm{d} v \mathrm{~d} \sigma \mathrm{d} \omega=K_{v} I_{v} \mathrm{~d} m \mathrm{~d} v \mathrm{~d} \omega,
$$

$\mathrm{d} m$ denoting the mass element, $\mathrm{d} v$ and $\mathrm{d} \omega$ are respectively the element of frequency and the elementary solid angle (Figure 1).

Introducing the phase function $p(\cos \theta)$ in the above expression, the rate of energy scattered in a direction making an angle $\theta$ with the incident beam through a solid angle $\mathrm{d} \omega$ ' is given by the relation:

$$
K_{v} I_{v} p(\cos \theta) \frac{\mathrm{d} \omega^{\prime}}{4 \pi} \mathrm{d} m \mathrm{~d} v \mathrm{~d} \omega
$$

The rate of energy lost by diffusion in all directions is then obtained by the integral:

$$
K_{v} I_{v} \mathrm{~d} m \mathrm{~d} v \mathrm{~d} \omega \int p(\cos \theta) \frac{\mathrm{d} \omega^{\prime}}{4 \pi}
$$

\section{- The emission component.}

The emission coefficient makes it possible to define the fraction of electromagnetic energy with a frequency between $v$ and $v+\mathrm{d} v$ emitted by a mass $\mathrm{dm}$ in directions confined in an elementary solid angle $\mathrm{d} \omega$ during the time $\mathrm{d} t$ by:

$$
j_{v} \mathrm{~d} m \mathrm{~d} v \mathrm{~d} \omega \mathrm{d} t
$$

This energy emitted by the material takes into account the fraction emitted by scattering of an incident radiation which can be expressed as a function of the angles $(\mathcal{\vartheta}, \varphi)$ and $\left(\mathcal{V}, \varphi^{\prime}\right)$ which respectively characterize the directions of the incident and scattered radiation and given by the expression:

$$
K_{v} \mathrm{~d} m \mathrm{~d} v \mathrm{~d} \omega p\left(\vartheta, \varphi, \vartheta^{\prime}, \varphi^{\prime}\right) I_{v}\left(\vartheta^{\prime}, \varphi^{\prime}\right) \frac{\sin \vartheta^{\prime} \mathrm{d} \vartheta^{\prime} \mathrm{d} \varphi^{\prime}}{4 \pi}
$$

The contribution of the diffusion to the emission coefficient noted $j_{v}^{(s)}$ being: 


$$
j_{v}^{(s)}(\vartheta, \varphi)=K_{v} \frac{1}{4 \pi} \int_{0}^{\pi} \int_{0}^{2 \pi} p\left(\vartheta, \varphi, \vartheta^{\prime}, \varphi^{\prime}\right) I_{v}\left(\vartheta^{\prime}, \varphi^{\prime}\right) \sin \vartheta^{\prime} \mathrm{d} \vartheta^{\prime} \mathrm{d} \varphi^{\prime}
$$

For an essentially diffusing atmosphere, we have an equality between $j_{v}$ and $j_{v}^{(s)}$,

In the case of an atmosphere in local thermodynamic equilibrium, at the local temperature $T$, the emission coefficient is given by Kirchhoff s law according to the equation:

$$
j_{v}=K_{v} B_{v}(T), \text { avec } B_{v}(T)=\frac{2 h v^{3}}{c^{2}} \frac{1}{\mathrm{e}^{h v / k T}-1},
$$

$B_{v}(T)$ is the Planck's function, $k$ the Boltzmann's constant and $h$ the Planck's constant.

\section{- The source function.}

The source function is defined by the ratio of the emission coefficient to that of absorption. According to the definitions of these coefficients formulated in Equations (2) and (6), we obtain for a diffusing atmosphere:

$$
\mathfrak{I}_{v}(\vartheta, \varphi)=\frac{1}{4 \pi} \int_{0}^{\pi} \int_{0}^{2 \pi} p\left(\vartheta, \varphi, \vartheta^{\prime}, \varphi^{\prime}\right) I_{v}\left(\vartheta^{\prime}, \varphi^{\prime}\right) \sin \vartheta^{\prime} \mathrm{d} \vartheta^{\prime} \mathrm{d} \varphi^{\prime}
$$

In the case of a local equilibrium of the atmosphere we have: $\mathfrak{J}_{v}=B_{v}(T)$.

\section{- The radiative transfer equation for parallel plane atmospheres.}

This fundamental equation is the expression of the variation in intensity of a radiation when passing through a medium characterized by an absorption coefficient $K_{v}$ and an emission coefficient $j_{v}$ It is an integrodifferential equation which takes into account the losses and gains of energy that occur during the crossing and can be written:

$$
\frac{\mathrm{d} I_{v}}{\mathrm{~d} s}=-K_{v} \rho \cdot I_{v}+j_{v} \rho
$$

From which we get:

$$
-\frac{\mathrm{d} I_{v}}{K_{v} \rho \mathrm{d} s}=I_{v}-\mathfrak{J}_{v}
$$

The radiative transfer equation can have different expressions that are more or less complex depending on the type of atmosphere, the symmetry of the problem and the coordinate system used.

For example, in the case of parallel plane atmospheres, the transfer equation can be expressed as a function of the normal optical thickness $\tau=\int_{\varepsilon}^{\infty} K \rho \mathrm{d} z$ and takes the following standard form:

$$
\mu \frac{\mathrm{d} I(\tau, \mu, \varphi)}{\mathrm{d} \tau}=I(\tau, \mu, \varphi)-\Im(\tau, \mu, \varphi)
$$

where $\mu=\cos \theta$.

In the case of semi-finished parallel plane atmospheres (Figure 2), we introduce the functions relating to diffuse reflection $S$ and transmission $T$ such as: 


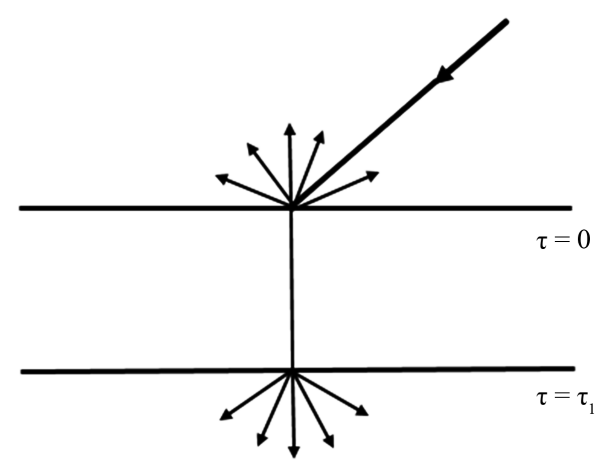

Figure 2. Illustration of diffusion and transmission in the case of a parallel atmosphere.

$$
\begin{gathered}
I(0, \mu, \varphi)=\frac{F}{4 \mu} S\left(\tau_{1}, \mu, \varphi, \mu_{0}, \varphi_{0}\right) \\
I\left(\tau_{1},-\mu, \varphi\right)=\frac{F}{4 \mu} T\left(\tau_{1}, \mu, \varphi, \mu_{0}, \varphi_{0}\right), \quad 0 \leq \mu \leq 1
\end{gathered}
$$

The fundamental transfer equation can then be written [14]:

$$
\begin{aligned}
& \mu \frac{\mathrm{d} I(\tau,-\mu, \varphi)}{\mathrm{d} \tau} \\
& =I(\tau, \mu, \varphi)-\frac{1}{4 \pi} \int_{-1}^{1} \int_{0}^{2 \pi} p\left(\mu, \varphi, \mu^{\prime}, \varphi^{\prime}\right) I\left(\tau, \mu^{\prime}, \varphi^{\prime}\right) \mathrm{d} \mu^{\prime} \mathrm{d} \varphi^{\prime} \\
& -\frac{1}{4} F \mathrm{e}^{-\tau / \mu_{0}} p\left(\mu, \varphi,-\mu_{0}, \varphi_{0}\right)
\end{aligned}
$$

\section{The Game Code}

The GAME code "Global Atmospheric Model" is a model inspired by the work of Dubuisson et al. [11], and has been adapted in a treatment chain to compute the aerosol radiative forcing [12]. This model takes into account the absorption and diffusion processes by gases (molecules) and particles in atmosphere, according to the approximation of an atmosphere cut into 33 planar parallel layers. In accordance with the description formulated by Roger et al. [12] the absorption takes into account the absorbers of the short wave band of the electromagnetic spectrum and is based on the results of an execution code constructed according to the line by line method [15], while the problems of multiple scattering are treated by the discrete ordinate method by which the entire solar spectrum is covered with a step of $100 \mathrm{~cm}^{-1}$, which gives in wavelength a variable step defined by $\Delta \lambda$ $=\lambda^{2} / 100$. In this method, the phase functions and the radiance are decomposed into Legendre polynomials, the interactions between the multiple diffusions and the gas absorption are treated by the use of the correlated k-method [16].

The modified GAME code incorporates the combined effects of:

- Absorption by gases, $\mathrm{H}_{2} \mathrm{O}, \mathrm{O}_{2}, \mathrm{CO}_{2}, \mathrm{O}_{3}$,

- Rayleigh scattering by molecules,

- Absorption by aerosols through the simple diffusion albedo,

- Diffusion due to aerosols by the asymmetry factor, 
- The surface albedo.

The modified GAME code gives the output of the simulation, the values of the upward and downward radiative fluxes from which the radiative forcings at the top of the atmosphere, within the atmosphere, and at the surface are obtained, as well as the rate of heating.

Direct radiative forcing to the earth's surface is obtained from the net surface flux taking into account the aerosol layer $F_{B O A}^{a}$ and the net surface flux calculated without the effect of aerosols $F_{B O A}^{0}$. This direct radiative forcing $\triangle F_{B O A}$ is then:

$$
\Delta F_{B O A}=F_{B O A}^{a}-F_{B O A}^{0}
$$

Likewise, the direct radiative forcing at the top of the atmosphere is obtained by:

$$
\Delta F_{T O A}=F_{T O A}^{a}-F_{T O A}^{0}
$$

Atmospheric radiative forcing $\Delta F_{A T M}$ is a simple difference between radiative forcing at the top of the atmosphere and at the earth's surface according to the relationship:

$$
\Delta F_{A T M}=\Delta F_{T O A}-\Delta F_{B O A}
$$

The vertical profile of the determined heating rate is calculated from the following thermodynamic relationship [12]:

$$
\frac{\partial T}{\partial t}=-\frac{1}{\rho C_{p}} \frac{\partial F(z)}{\partial z}
$$

where: $T$ denotes the air temperature, and $t$ the time,

$\rho$ is the density of the atmosphere,

$C_{p}$ is the specific heat of the air,

$F(z)$ is the net flux at altitude $z$.

To obtain the results mentioned above, you need several data sets as input to the modified GAME code. These include:

- Optical properties: optical thicknesses, asymmetry parameter, single scattering albedo and Angström exponent were used as input to the simulation. In this study, we used the monthly averages of AERONET (Aerosol Robotic Network) level 2 measurement and inversion data from 1999 to 2006 [17] [18],

- The vertical temperature and humidity profiles were obtained by radiosonde measurements from the earth's surface to an altitude of more than 25000 meters,

- The daily evolution of ambient temperature obtained by synoptic measurements averaged per month,

- The Earth's surface albedo estimated for the entire solar spectrum from the MODIS (Moderate-resolution Imaging Spectroradiometer) sensor,

- The solar angle, for the daily representation. 


\section{Results}

The monthly average values of the modified GAME code input data were used to simulate the radiative impact of aerosols at the top of the atmosphere, in the atmospheric layer and on the earth's surface, month by month. The results are presented in Table 1.

These results show an overall cooling effect at the top of the atmosphere due to the backscattering in space of solar radiation, a warming in the atmospheric column which is explained by the absorption of radiation by anthropogenic pollutants and again a cooling at the earth's surface resulting from the attenuation of radiation as it passes through the aerosol layer. These effects are shown by negative values of radiative forcing at the top of the atmosphere ranging from $-6.77 \mathrm{~W} / \mathrm{m}^{2}$ to $-2.56 \mathrm{~W} / \mathrm{m}^{2}$ and at the surface (from $-41.00 \mathrm{~W} / \mathrm{m}^{2}$ to -21.68 $\mathrm{W} / \mathrm{m}^{2}$ ), as well as positive values of the forcing in the atmosphere. This situation reflects a decrease in the net flux received at the ground and at the top, and an increase in the flux of the atmosphere.

In accordance with what one might expect from the analysis of optical properties in connection with climate dynamics, the radiative forcing at the surface is greater in the month of March-April when the calculation gives the lowest values between the months of August-September and November-December.

At the top of the atmosphere, the results are more subject to the uncertainties of the assumptions adopted. However, we note that the simulations indicate the lowest impacts in the period December-January-February and the highest in the period March-April-June as well as in October. The dynamics of the local climate cannot only justify this distribution of values. We see through these values the effect of large-scale dynamics and the combined impact of the seasonal succession in the North (winter, summer ...) and in the South (hot, rainy season ...).

The differences between the values of the impacts at the top of the atmosphere and at the surface logically induce a significant impact from the aerosols in the atmospheric layer (Figure 3).

Table 1. Simulation of the radiative impact at the surface (BOA), in the atmospheric layer (ATM), at the top of the atmosphere (TOA) and warming $(\Delta \mathrm{T})$ of the first layer.

\begin{tabular}{ccccc}
\hline & BOA_IMPACT & ATM_IMPACT & TOA_IMPACT & $\Delta$ T_1st_layer \\
\hline January & -34.3 & 31.7 & -2.5 & 1.7 \\
February & -30.9 & 27.2 & -3.6 & 1.5 \\
March & -41.0 & 34.7 & -6.2 & 1.8 \\
April & -35.8 & 31.3 & -4.5 & 1.7 \\
May & -33.6 & 27.5 & -6.0 & 1.5 \\
June & -32.9 & 26.1 & -6.7 & 1.4 \\
July & -28.6 & 25.4 & -3.2 & 1.4 \\
August & -21.6 & 15.3 & -6.3 & 0.8 \\
September & -24.6 & 20.5 & -4.1 & 1.2 \\
October & -27.0 & 20.2 & -6.8 & 1.1 \\
November & -23.4 & 18.7 & -4.6 & 1.0 \\
December & -24.9 & 22.3 & -2.5 & 1.3 \\
\hline
\end{tabular}




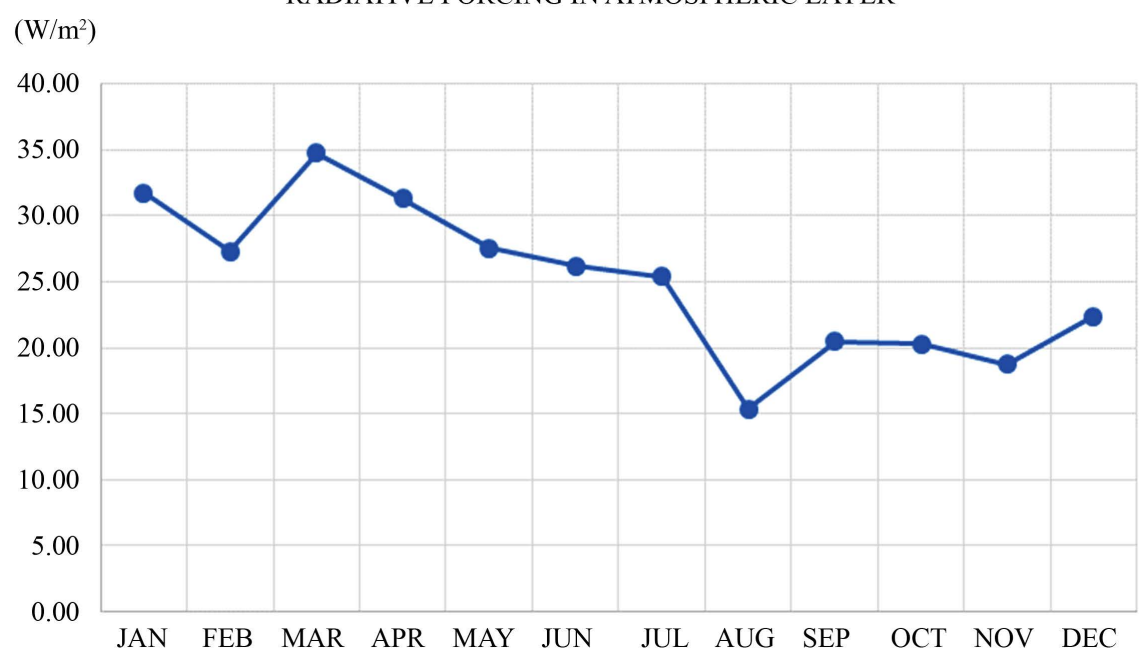

Figure 3. Simulation of the radiative impact in the atmospheric layer.

Figure 3 clearly shows a maximum impact in March-April, a period when the obtained values are greater than $30 \mathrm{~W} / \mathrm{m}^{2}\left(34.74 \mathrm{~W} / \mathrm{m}^{2}\right.$ in March and 31.30 $\mathrm{W} / \mathrm{m}^{2}$ in April) and a minimum impact in August estimated at $15.38 \mathrm{~W} / \mathrm{m}^{2}$. A connection can be made with the characteristic seasonal succession of the climate in West Africa and the Sahel. In fact, the month of August is in the rainy season, characterized by the preponderance of humid monsoon winds from the South. It is during this period that the suspension of particles is the least due to the frequency of the rains responsible for the leaching of mineral dust. The obtained values reflect the importance of pollution aerosols and their contribution, which constitutes the bulk of the forcing calculated in August, but also the contribution of dust of local and Saharan origin. It is this impact that is shown in a difference of up to nearly $20 \mathrm{~W} / \mathrm{m}^{2}$ between the values calculated in March and in August. As shown in Figure 3, the atmospheric impact decreases from Mars to a minimum in August before increasing from September to February. It is at this time that the rainy season ends, the end of which is marked by the gradual installation of harmattan winds from the North and charged with dust from the Sahara desert.

The atmospheric impact can be reflected by a change in temperature. The simulation indicates a warming of the first layer of the atmosphere (this is the layer in contact with the earth) whose temperature variation is relatively large and ranges from $0.83^{\circ} \mathrm{C}$ in August to about $1.8^{\circ} \mathrm{C}$ in Mars as shown in Figure 4.

\section{Discussion}

Radiative forcing due to aerosols is poorly characterized in climate models [19]. In particular, the calculation of radiative impact in the West African region, specifically at the scale of an urban area, is very poorly documented in literature. Numerous studies carried out generally give an average indication of the radiative impact in the entire region, which does not allow us to distinguish the 


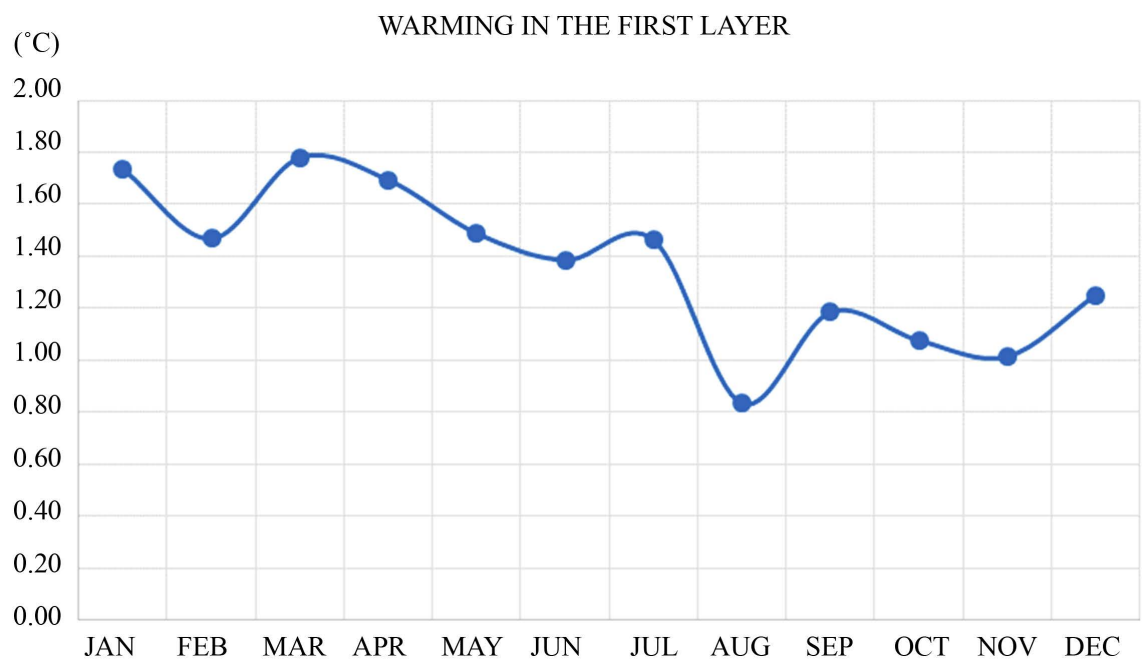

Figure 4. Evolution of the average monthly warming in the first layer.

particular contribution of an urban site. Some studies conducted at the scale of an urban area have been carried out but in places geographically distant and climatically different from our study site. We can cite certain studies such as ESCOMPTE [12] [20] carried out in the city of Marseille, MINOS in the island of Crete in Greece.

In West Africa, simulation work of the radiative impact of mineral dust as well as its effect on the variability of precipitation during the monsoon period has been carried out [21]. This simulation made of a coupling of meteorological model and the aerosol/chemistry model (WRF-Chem) gave results summarized in Figure 5 for short (SW) and long (LW) wavelengths as well as for the entire spectrum (Net) at top of the atmosphere ((a), (b), (c)), at the surface ((g), (h), (i)) and within the atmospheric layer ((d), (e), (f)).

Figure 5 shows that in the West Africa region South of the Sahara, the direct impact of aerosols results in cooling at the top of the atmosphere and on the earth's surface, followed by warming in the atmosphere layer for short wavelengths and for the whole spectrum. For long wavelengths, the opposite effect is observed. The simulation indicates for short wavelengths radiative forcing values ranging from $-3 \mathrm{~W} / \mathrm{m}^{2}$ to $-10 \mathrm{~W} / \mathrm{m}^{2}$ at the top of the atmosphere. In the atmospheric layer, warming results in a positive radiative forcing of between 10 $\mathrm{W} / \mathrm{m}^{2}$ and $30 \mathrm{~W} / \mathrm{m}^{2}$. At the earth's surface, we see that the region covered by our study presents a radiative forcing of between $-15 \mathrm{~W} / \mathrm{m}^{2}$ and $-30 \mathrm{~W} / \mathrm{m}^{2}$. These values show a very good agreement with the results of our simulation which gave for the same spectral band, the monthly mean values of the radiative forcing very similar. Our calculations for every month of the year gave values between -2.5 and $-6.77 \mathrm{~W} / \mathrm{m}^{2}$ at the top of the atmosphere, between -21 and $-41 \mathrm{~W} / \mathrm{m}^{2}$ at the surface and between 15.38 and $34.74 \mathrm{~W} / \mathrm{m}^{2}$ in the atmospheric layer. The values of the forcing at the top of the atmosphere presented by our simulation are consistent with the global mean value of the direct effect of aerosols in the absence of clouds, obtained from satellite observations and estimated at $-5.5 \pm 0.2 \mathrm{~W} / \mathrm{m}^{2}$ 


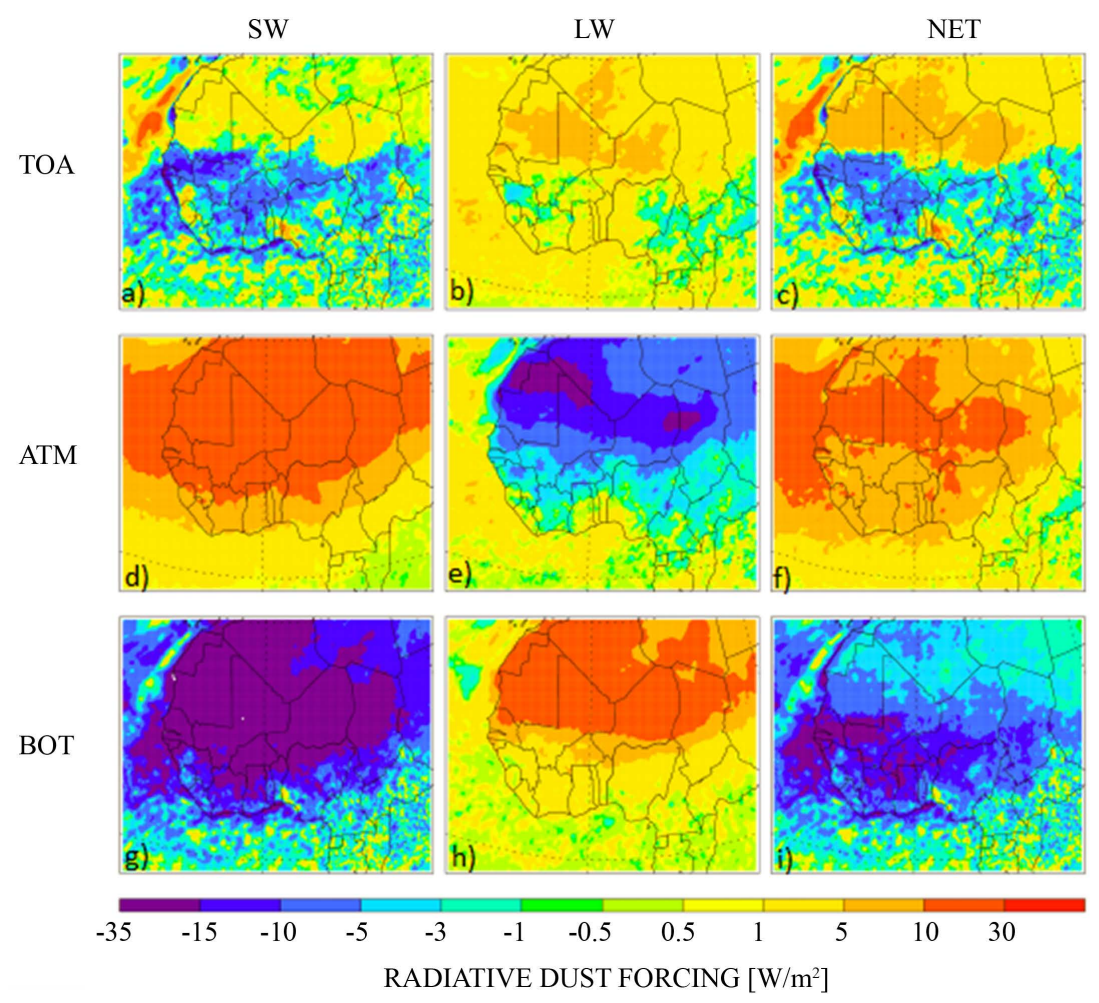

Figure 5. WRF-Chem simulations of radiative dust forcing in Africa for short wavelengths (SW), long wavelengths (LW) and for the short + long (Net), at the top of the atmosphere (TOA: (a), (b), (c)), in the atmosphere (ATM: (d), (e), (f)) and at the surface (BOA: (g), (h), (i))-Extract from C. Zhao et al., 2011 [21].

above oceans and $4.9 \pm 0.7 \mathrm{~W} / \mathrm{m}^{2}$ above land [1] [22]. Likewise, using an estimation method based on CERES and MODIS observations, [23], estimates the average value of the global direct impact of aerosols at the top of the atmosphere at $-3.1 \pm 1.0 \mathrm{~W} / \mathrm{m}^{2}$ in cloudy sky conditions compared to $7.3 \pm 1.6 \mathrm{~W} / \mathrm{m}^{2}$ in clear sky conditions.

The results are also in good agreement with those of a simulation of the radiative effects of dust carried out in Africa as part of the AMMA program, following the SOP plane measurement campaign over Benin and Niger on the 13th and June 14, 2006. This simulation which used the STREAMER radiative calculation model estimated these radiative effects by a warming whose maximum is between the daily values of $1.5 \mathrm{~K} /$ day and $3 \mathrm{~K} /$ day [24] while our calculation estimates this magnitude between $0.83^{\circ} \mathrm{C}$ and $1.78^{\circ} \mathrm{C}$. As part of the same program, various simulations of the direct radiative impact of aerosols in the period December 2005 to February 2006 were carried out during the DABEX campaign. They gave for the short and long wavelength group, in the Sahelian band, the results presented in Table 2 [25].

According to other radiative forcing calculations such as those carried out by Mallet et al. [26] direct radiative forcing values at the surface and the top of the atmosphere of respectively $-57.9 \mathrm{~W} / \mathrm{m}^{2} /$ AOT550 $\mathrm{nm}$ and $-17.5 \mathrm{~W} / \mathrm{m}^{2} /$ AOT550 $\mathrm{nm}$ in Djougou in the period from January 17 to 24,2006 have been reported. 
Table 2. Radiative forcing over the Sahel $\left(\mathrm{W} / \mathrm{m}^{2}\right)$.

\begin{tabular}{cccc}
\hline & \multicolumn{3}{c}{ Sahel: $\left(5^{\circ} \mathrm{N} ; 13^{\circ} \mathrm{N}\right)\left(10^{\circ} \mathrm{W} ; 20^{\circ} \mathrm{E}\right)$} \\
\cline { 2 - 4 } & SRF & ATM & TOA \\
\hline REF & -28.27 & 24.4 & -3.87 \\
DUSTex & -5.58 & 2.61 & -2.97 \\
BCOCex & -23.04 & 21.99 & -1.06 \\
\hline
\end{tabular}

The REF simulation takes into account all the aerosols, the DUSTex simulation concerns only mineral dust and the BCOCex simulation, carbonaceous aerosols [25].

The set of radiative forcing values carried out according to different approaches in the region clearly shows that the order of magnitude of the radiative impacts that we have found is appreciable even if it should be noted that the context, the period or the methodology used are the origin of some differences that we have seen.

\section{Conclusion}

This work is a simulation exercise which follows a climatological study of the optical properties of aerosols in Ouagadougou. This study made it possible to characterize the optical and microphysical properties and showed the strong correlation with the seasonal succession of the local climate [27]. Similarly, the results of the radiative forcing calculation logically showed the influence of the dynamics of the Harmattan and monsoon winds that alternate in West Africa. At the top of the atmosphere, in the atmospheric layer as well as on the surface, the extreme values were observed in March and August. It should be noted that the month of March is in the midst of the dry season and represents the period of large desert aerosol emissions carried by the harmattan winds and the month of August corresponds to the wettest month of the rainy season which runs from June to October. We can see in these results the persistent effect of anthropogenic emissions linked to human activity but also the marked seasonal effect of dust from the Sahara which, although recognized to be mainly diffusing, contributes significantly to the radiation balance of Sahelian cities such as Ouagadougou.

\section{Acknowledgements}

The authors are grateful to International Science Program (ISP), Uppsala University for their support to BUF 01 research group and to this work.

\section{Conflicts of Interest}

The authors declare no conflicts of interest regarding the publication of this paper.

\section{References}

[1] Intergovernmental Panel on Climate Change (2007) Climate Change 2007: The 
Physical Science Basis. Intergovernmental Panel on Climate Change, Geneva.

[2] Kiehl, J.T., Schneider, T.L., Rasch, P.J., Barth, M.C. and Wong, J. (2000) Radiative Forcing Due to Sulfate Aerosols from Simulations with the National Center for Atmospheric Research Community Climate Model, Version 3. Journal of Geophysical Research: Atmospheres, 105, 1441-1457. https://doi.org/10.1029/1999JD900495

[3] Haywood, J. and Boucher, O. (2000) Estimates of the Direct and Indirect Radiative Forcing Due to Tropospheric Aerosols: A Review. Reviews of Geophysics, 38, 513-543. https://doi.org/10.1029/1999RG000078

[4] Hansen, J., Sato, M. and Ruedy, R. (1997) Radiative Forcing and Climate Response. Journal of Geophysical Research, 102, 6831-6864.

https://doi.org/10.1029/96JD03436

[5] Rosenfeld, D. (1999) TRMM Observed First Direct Evidence of Smoke from Forest Fires Inhibiting Rainfall. Geophysical Research Letters, 26, 3105-3108. https://doi.org/10.1029/1999GL006066

[6] Twomey, S. (1974) Pollution and the Planetary Albedo. Atmospheric Environment, 8, 1251-1256. https://doi.org/10.1016/0004-6981(74)90004-3

[7] Twomey, S. (1977) The Influence of Pollution on the Shortwave Albedo of Clouds. Journal of the Atmospheric Science, 34, 1149-1152. https://doi.org/10.1175/1520-0469(1977)034<1149:TIOPOT>2.0.CO;2

[8] Coakley, J.A., Cess, R.D. and Yurevich, F.B. (1983) The Effect of Tropospheric Aerosols on the Earth's Radiation Budget: A Parameterization for Climate Models. Journal of the Atmospheric Science, 40, 116-138. https://doi.org/10.1175/1520-0469(1983)040<0116:TEOTAO >2.0.CO;2

[9] Pincus, R. and Baker, M. (1994) Effect of Precipitation on the Albedo Susceptibility of Clouds in Marine Boundary Layers. Nature, 372, 250-252. https://doi.org/10.1038/372250a0

[10] Albrecht, B.A. (1989) Aerosols, Clouds Microphysics, and Fractional Cloudiness. Science, 245, 1227-1230. https://doi.org/10.1126/science.245.4923.1227

[11] Dubuisson, P., Buriez, J.C. and Fouquart, Y. (1996) High Spectral Resolution Solar Radiative Transfer in Absorbing and Scattering Media: Application to the Satellite Simulation. Journal of Quantitative Spectroscopy \& Radiative Transfer, 55, 103-126. https://doi.org/10.1016/0022-4073(95)00134-4

[12] Roger, J.-C., Mallet, M., Dubuisson, P., Cachier, H., Vermote, E., Dubovik, O. and Despiau, S. (2006) A Synergic Approach for Estimating the Local Direct Aerosol Forcing: Application to an Urban Zone during the Experience sur Site Pour Contraindre les Modèles de Pollution et de Transport d'Emission (ESCOMPE) Experiment. Journal of Geophysical Research: Atmospheres, 111, Article ID: D13208. https://doi.org/10.1029/2005JD006361

[13] Korgo, B. (2014) Optical and Microphysical Characterization of Atmospheric Aerosol in West African Urban Areas: Application to Radiative Forcing Calculations in Ouagadougou. PhD Thesis, University Blaise Pascal, Clermont Ferrand, France/University of Ouagadougou, Burkina Faso.

[14] Chandrasekhar, S. (1960) Radiative Transfer. Dovers Publications, Mineola.

[15] Scott, N.A. (1974) A Direct Method of Computation of the Transmission Function of an Inhomogeneous Gaseous Medium-I: Description of the Method. Journal of Quantitative Spectroscopy and Radiative Transfer, 14, 691-704. https://doi.org/10.1016/0022-4073(74)90116-2

[16] Lacis, A.A. and Oinas, V. (1991) A Description of the Correlated K-Distribution 
Method. Journal of Geophysical Research: Atmospheres, 96, 9027-9064. https://doi.org/10.1029/90JD01945

[17] Holben, B.N., Eck, T.F., Slutsker, I., Tanre, D., Buis, J.P., Setzer, A., Vermote, E., Reagan, J.A., Kaufman, Y., Nakajima, T., Lavenu, F., Jankowiak, I. and Smirnov, A. (1998) AERONET - A Federated Instrument Network and Data Archive for Aerosol Characterization. Remote Sensing of Environment, 66, 1-16. https://doi.org/10.1016/S0034-4257(98)00031-5

[18] Dubovik, O., Holben, B.N., Eck, T.F., Smirnov, A., Kaufman, Y.J., King, M.D., Tanré, D. and Slutsker, I. (2002) Variability of Absorption and Optical Properties of Key Aerosol Types Observed in Worldwide Locations. Journal of Atmospheric Sciences, 59, 590-608. https://doi.org/10.1175/1520-0469(2002)059<0590:VOAAOP >2.0.CO;2

[19] Saha, A., Mallet, M., Roger, J.-C., Dubuisson, P., Piazzola, J. and Despiau, S. (2008) One Year Measurement of Aerosol Optical Properties over an Urban Coastal Site: Effect on Local Direct Radiative Forcing. Atmospheric Research, 90, 195-202. https://doi.org/10.1016/j.atmosres.2008.02.003

[20] Mallet, M., Pont, V., Liousse, C., Roger, J.C. and Dubuisson, P. (2006) Simulation of Aerosol Radiative Properties with the Orisam-Rad Model during a Pollution Event (ESCOMPTE 2001). Atmospheric Environment, 40, 7696-7705. https://doi.org/10.1016/j.atmosenv.2006.08.031

[21] Zhao, C., Liu, X., Leung, L.R. and Hagos, S. (2011) Radiative Impact of Mineral Dust on Monsoon Precipitation Variability over West Africa. Atmospheric Chemistry and Physics, 11, 1879-1893. https://doi.org/10.5194/acp-11-1879-2011

[22] Yu, H., Kaufman, Y.J., Chin, M., Feingold, G., Remer, L.A., Anderson, T.L., Balkanski, Y., Bellouin, N., Boucher, O., Christopher, S., Decola, P., Kahn, R., Koch, D., Loeb, N., Schulz, M.S., Takemura, T. and Zhou, M. (2006) A Review of Measurement-Based Assessments of the Aerosol Direct Radiative Effect and Forcing. Atmospheric Chemistry and Physics, 6, 613-666.

https://doi.org/10.5194/acp-6-613-2006

[23] Oh, H.R., Choi, Y.S., Ho, C.H. and Jeong, M.J. (2013) Estimation of Aerosol Direct Radiative Effects for All-Sky Conditions from CERES and MODIS Observations. Journal of Atmospheric and Solar-Terrestrial Physics, 102, 311-320. https://doi.org/10.1016/j.jastp.2013.06.009

[24] Lemaître, C., Flamant, C., Cuesta, J., Rault, J.-C., Chazette, P., Formenti, P. and Pelon, J. (2010) Dust Aerosol Radiative Forcing over West Africa: A Case Study from the AMMA SOP. Geophysical Research Abstracts, 11, 1330.

[25] Malavelle, F., Pont, V., Mallet, M., Solmon, F., Johnson, B., Léon, J. and Liousse, C. (2011) Simulation of Aerosol Radiative Effects over West Africa during DABEX and AMMA SOP-0. Journal of Geophysical Research: Atmospheres, 116, Article ID: D08205. https://doi.org/10.1029/2010JD014829

[26] Mallet, M., Pont, V., Liousse, C., Gomes, L., Pelon, J., Osborne, S., et al. (2008) Aerosol Direct Radiative Forcing over Djougou (Northern Benin) during the African Monsoon Multidisciplinary Analysis Experiment (Special Observing Period-0). Journal of Geophysical Research: Atmospheres, 113, Article ID: D00C01. https://doi.org/10.1029/2007JD009419

[27] Korgo, B., Roger, J.-C. and Bathiebo, J. (2013) Climatology of Air Mass Trajectories and Aerosol Optical Thickness over Ouagadougou. Global Journal of Pure and Applied Sciences, 19, 169-181. 